\title{
Design of Servo System Based on TI DSP
}

\author{
Xinghong $\mathrm{Li}^{1, \mathrm{a}}$, Qian Xiang ${ }^{2, b}$ \\ ${ }^{1,2}$ The Engineering \& Technical college of Chengdu University of \\ Technology,Leshan,Sichuan,China \\ a545178279@qq.com, b1139916444@qq.com
}

Keywords: optoelectronic; track precision; Servo system

Abstract: O-E theodolite is a sort of complex and valuable instrument for measuring the parameter of moving target in the shooting range. While the track precision is the main technology target. The increasing complex and real-time arithmetic was applied in the servo system for boosting the track precision of O-E theodolite, so it demanded the servo controller must complete more and more operations in shorter time. While the actual O-E theodolite accomplished the capture and tracking etc. based on PC104 system, so designed the servo control system based on TMS320F2812 as CPU from the practicality, not only decreases the cubage of the system, but also increases the reliability and precision.

\section{Hardware structure}

Controllers applied for the servo system of theodolite presently are almost industrial computers with PC104 structure as showed in Fig.1. It accomplished the capture and track aim at moving target via leading the host computer, which needs lots of electronic boards to compose a set of successful controllers. So not only the cubage of a control system is increased, the reliability and precision undermined, but the cost is also increased more.

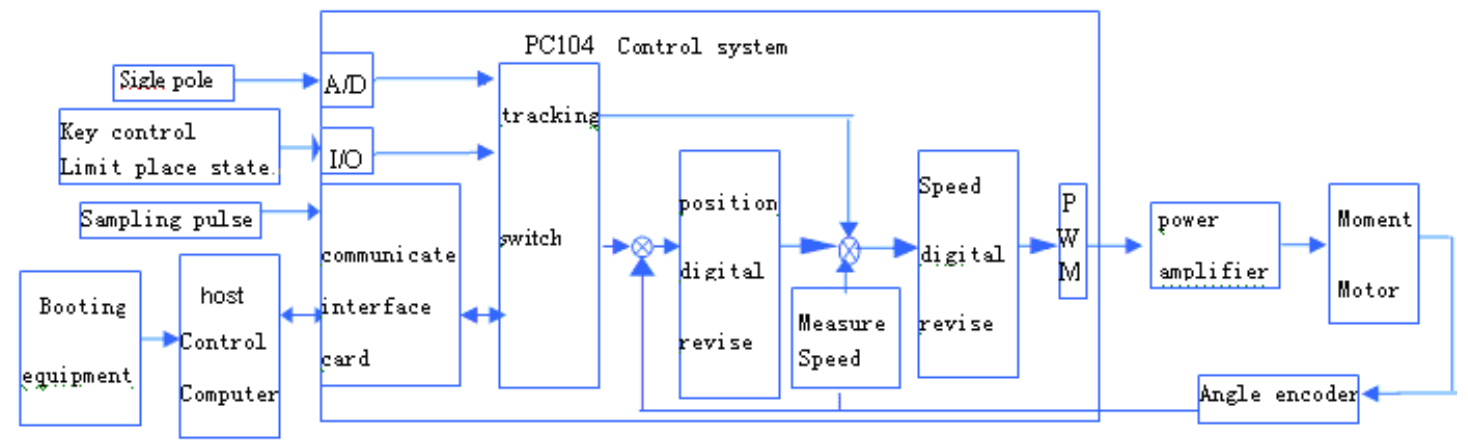

Fig.1 The servo controller of PC104 structure

A servo controller based on TMS320F2812 DSP from TI Corp. is designed for mending PC104 structure. Its well performances〔 $1 〕$ include 1500 MIPS for completing the operation of $32 * 32$ bit in single instruction cycle, high-powered ADC of 12 bit and the improved communicate ports, compatible code with TMS320C24X, convenient interface with photoelectric encoder etc. So it is possible that to substitute PC104 with DSP for servo controller as showed in Fig.2. It can satisfy the tracking system because of its obvious advantages. The controller includes motor, DSP CPU, the circuit of power conversion, the feedback circuit and some assistant circuits. 


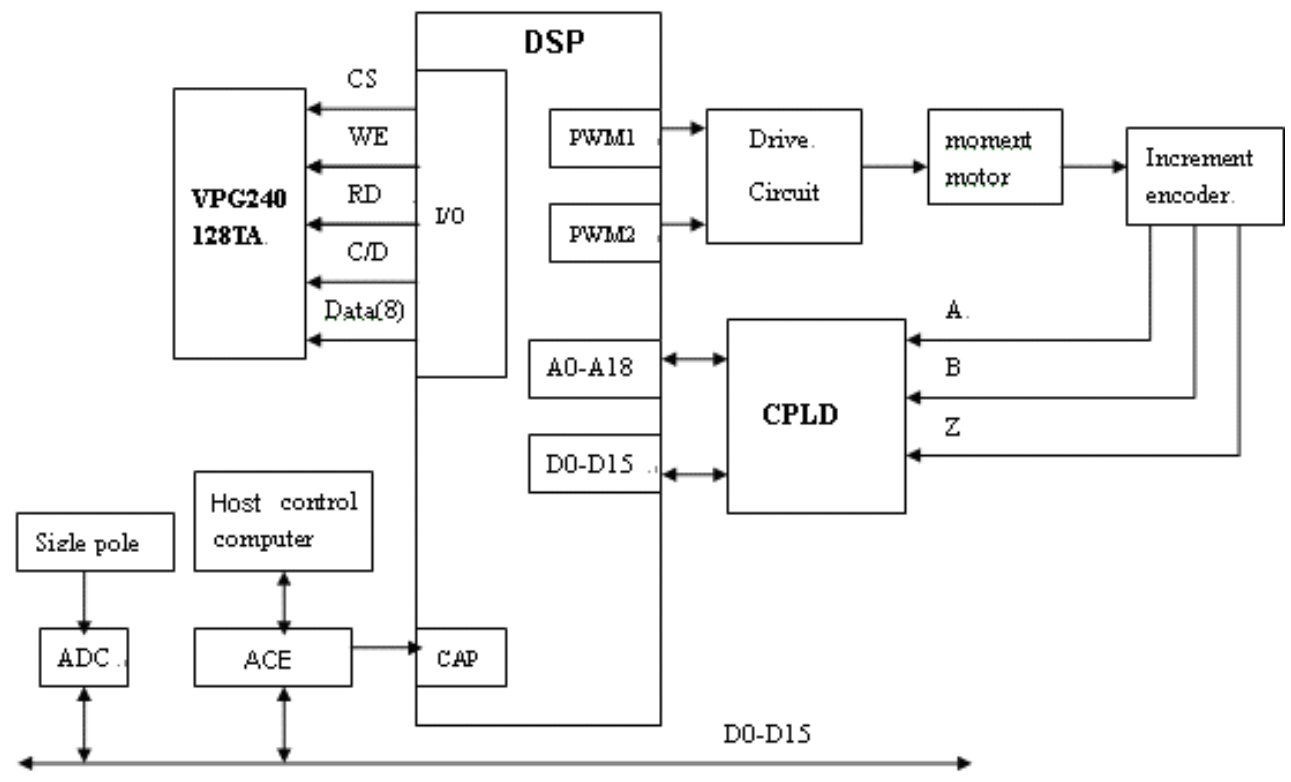

Fig.2 The servo controller of hardware structure based on TMS320F2812

DSP:TMS320F/C281X as the fresh blood of ZXXX series from TI is a sort of pointing DSP. It is applied in the digital control of the motor for its low cost, low power consumption, high performances. It integrates some advanced peripheral equipments, so it forms the genuine SCM.

ACE interface: DSP read the control command from the host control system via TL16C554. It cushions the data as 16 bits data before transmitting and receiving with FIFO mode, reduces CPU intermits and includes four improved TL16C550. Each channel completes the conversion of the series-parallel.

Circuit of PWM drive: TMS320F2812 has 16 PWM outputs for the motor. By controlling the duty cycle of PWM which determines the voltage of the motor, we can control the rotate speed of the motor. The servo motor is driven by PWM from DSP via IGBT which enhances the drive power of PWM. The circuit adopts PM100DSA120(IPM) which integrates IGBT and drive chip and it has the intact functions of insulate and protect.

Circuit of LCD display: The LCD module of control system displays some states for debug and self-check. The circuit adopts VPG240128TA-SC-HT-LED04 as LCD. Two 74LS245 chips switch $3.3 \mathrm{~V}$ to $5 \mathrm{~V}$ for drive $\mathrm{LCD}$ module.

\section{Software flow chart}

The feedback circuits based on the hardware circuit is built for improving the track precision of the control system. The feedback adjustor must disperse for the software programme, the software flow chart of arithmetic is showed in Fig.3.

\section{Servo adjustment}

PWM output via IGBT drive the servo motor and then drive the tracker running synchronously, getting $T_{m}$ from step response method, the model of the servo motor $G_{m}(S)=\frac{1 / K_{e}}{\left(T_{m} S+1\right)\left(T_{e} S+1\right)}$, adjust the tracking precision of a moving target which maximal angular 
velocity: $50^{\circ} / S$ and maximal angular acceleration: $30^{\circ} / S^{2}$, we can acquire the function of the equivalent sine moving target: $\theta_{i}=A_{m} \operatorname{Sin}\left(\omega_{i} t\right)$ 。

Here, two adjustment methods for the servo system are adopted.

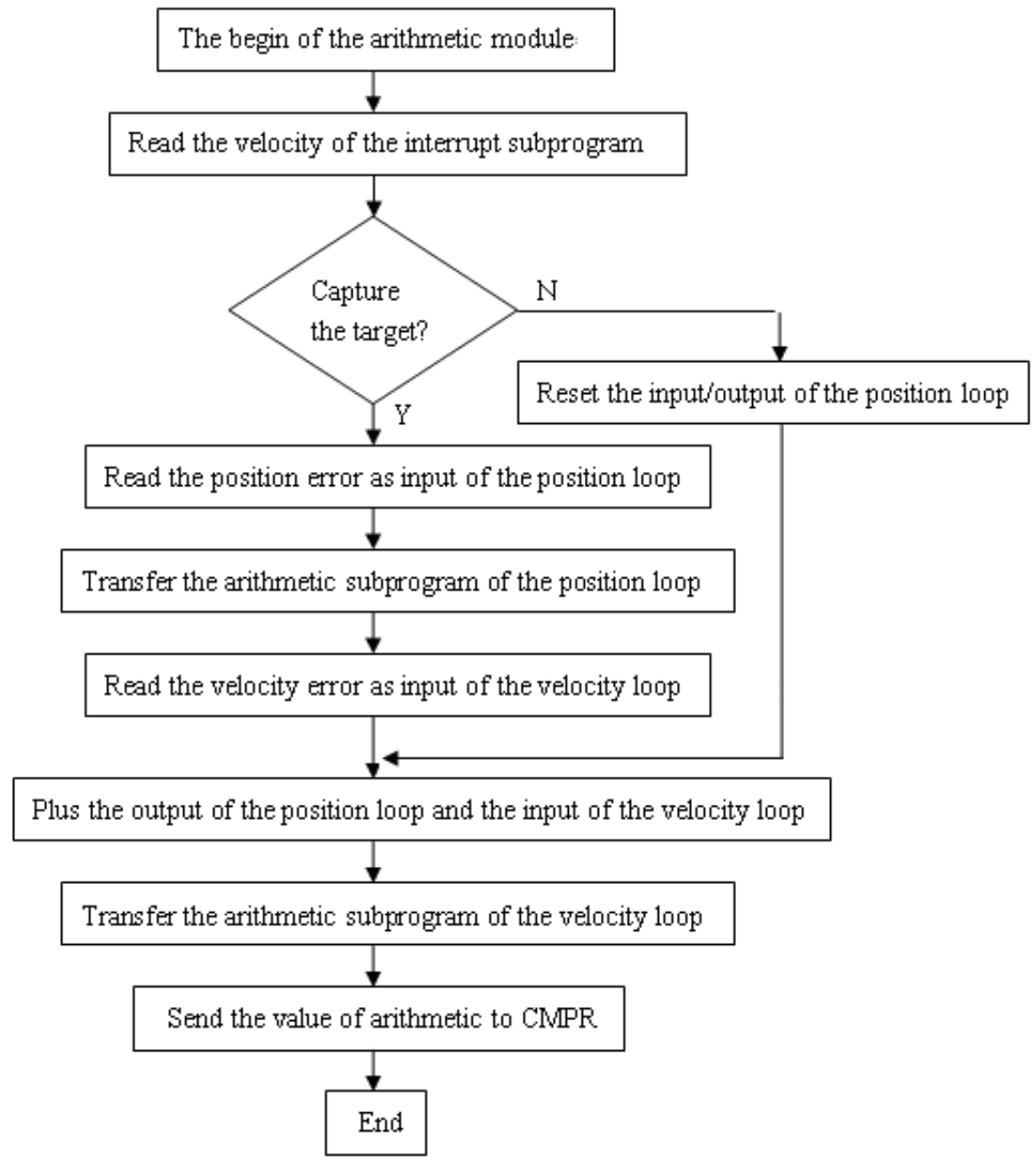

\section{The compound revise}

Fig.3 The arithmetic flow chart of control system

The compound control is to add an open control spur track which supply the velocity signal in close loop for improving the track precision.

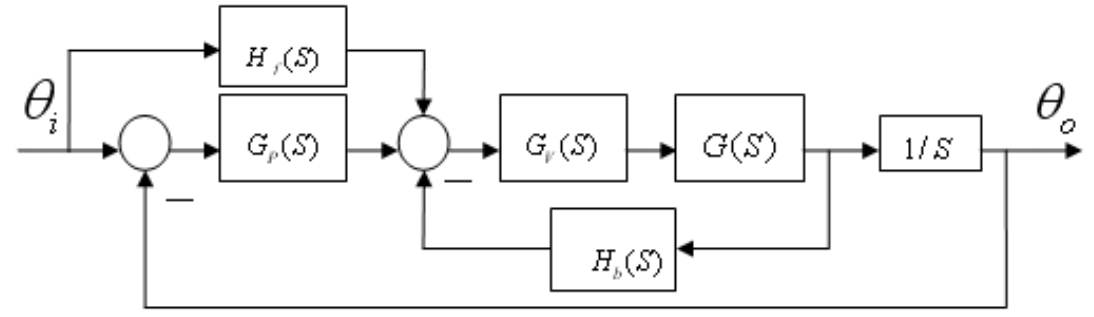

Fig4 The frame of compound revise

Here, the velocity regulator is $G_{V}(S)$ and the position regulator is $G_{P}(S) . G(S)$ is the transfer function of the control object, $H_{f}(S)$ is the transfer function of the feed-forward, $H_{b}(S)$ is the transfer function of speed feed-back. 
Getting $G_{V}(S)$ and $G_{P}(S)$ from Matlab/Simulink and the engineering experience with sampling rate of $800 \mathrm{~Hz}$. Using the stabilization criterion of the system, the leading-delay loop for the velocity adjustment and the leading-delay loop for the position adjustment: $G_{V}(S)=\frac{K_{1}\left(t_{1} S+1\right)\left(t_{2} S+1\right)}{\left(T_{1} S+1\right)\left(T_{2} S+1\right)}, G_{P}(S)=\frac{K_{2}\left(t_{3} S+1\right)\left(t_{4} S+1\right)}{\left(T_{3} S+1\right)\left(T_{4} S+1\right)}$

Velocity loop and position loop are very important in the servo control, because the testing precision effects on the precision of the motor controlling directly. Adopting the incremental photoelectric encoder can detect not only the rotate speed and the veer of the motor, but also the position of the stator relative to the rotor. An incremental encoder and the servo motor are mounted on a common axis, and then the encoder reading of servo motor is easily obtained using the QEP circuits.

QEP builted-in DSP can capture the rising and falling edge of the pulses, QEP based on the sequence of two pulses can distinguish the veer of the motor from Fig.5. DIR+ indicates the motor is clockwise while DIR- indicates the motor is counter-clockwise. DIR+ has output when A exceed B 1/4 period while DIR- has not outputs, which simplifies the systemic hardware.

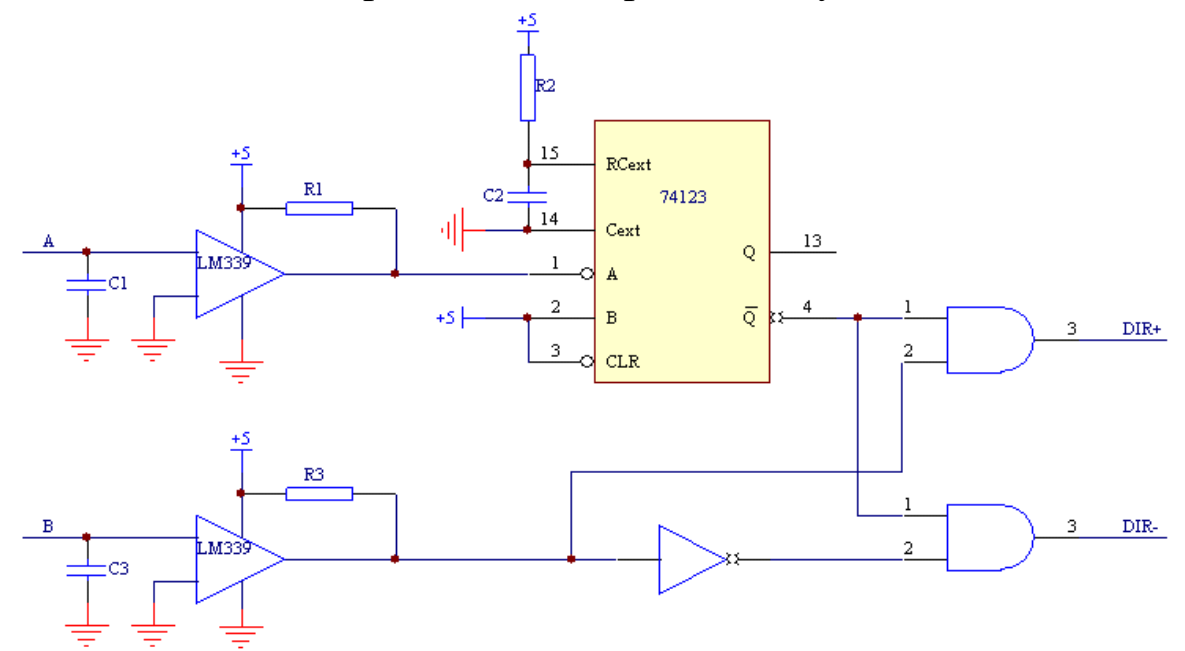

Fig.5 Circuit of DIR phase demodulation and shaping design

Four CAP pins builted-in DSP are defined as QEP mode with software. The two outputs, A pulse and B pulse with 90。 difference from the incremental encoder input QEP1 and QEP2, CAP circuit receives the two inputs and produces a quadrupled clock signal (CLK) which increases the precision and a direction signal (DIR).

Velocity and acceleration delay compensation adjustment:

The velocity and acceleration delay compensation adjustments are adopted to form as approximate equivalent compound control system.

Equivalent the velocity adjustment closed loop as a one-step inertia loop when the bandwidth of inner loop exceed five times than the bandwidth of outer loop: $G_{V}^{\prime}(S)=\frac{1}{\left(T_{s} S+1\right)}$. Here, velocity delay compensation loop: $\quad G_{V D}(S)=\frac{\alpha}{T_{\alpha} S+1}$; acceleration delay compensation loop: $G_{A D}(S)=\frac{\beta S}{T_{\beta} S+1}$ 。 


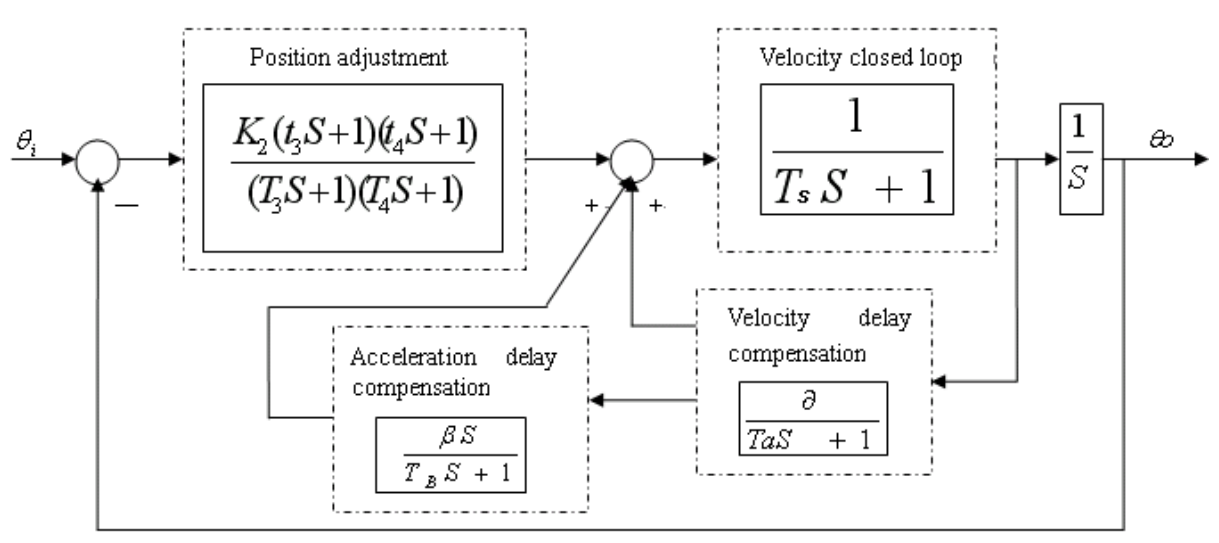

Fig.6 Principal diagram of equivalent compound control system

\section{metrical curve}

This system adopts the increment encoder as the feedback equipment. Receiving the signal of host control computer as the deliver signal, the speed of motor is different because the different deliver signal, using the compound revise make the motor obtain the desired speed, the following figures show the error curve and response curve.

When the code value of the received signal is 150 , we can numerate the speed of motor from the formula of the increment encoder: $N=\frac{M_{1}}{T_{C} * Z}$, the speed of motor is $6.25 \mathrm{circle} / \mathrm{s}$. The maximal error code is \pm 1 and the error is $0.67 \%$ after stabilization.

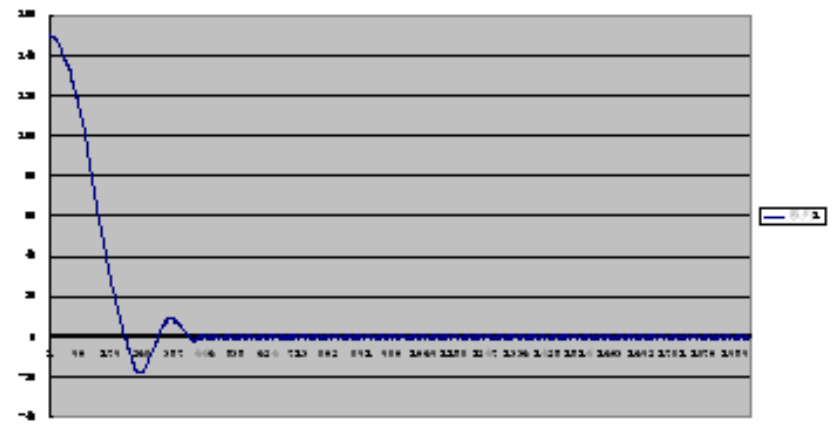

Fig.7 the code value of the received signal is 150 , the speed error curve after revise

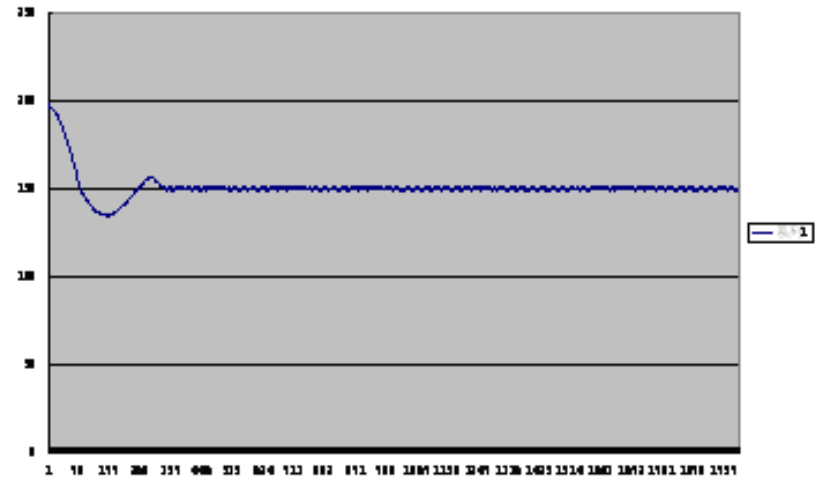

Fig.8 the code value of the received signal is 150 , the response curve of the motor after revise When the code value of the received signal is 250 , the speed of motor is 10.4 circle/s. The maximal error is code \pm 1 and the error is $0.4 \%$ after stabilization. 


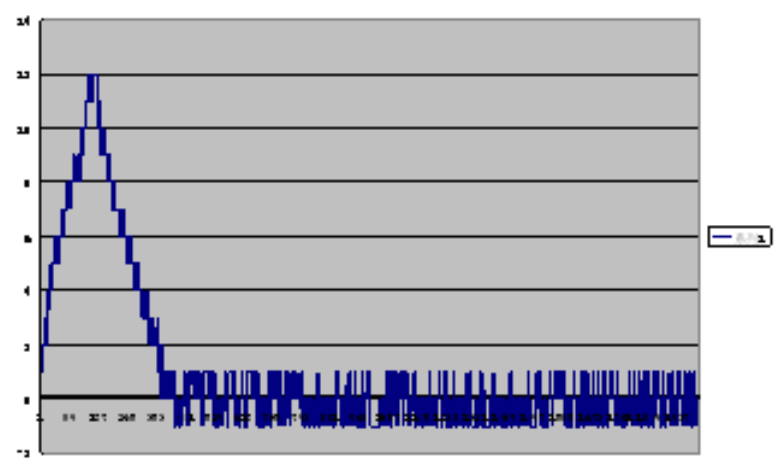

Fig.9 the code value of the received signal is 250 , the speed error curve after revise

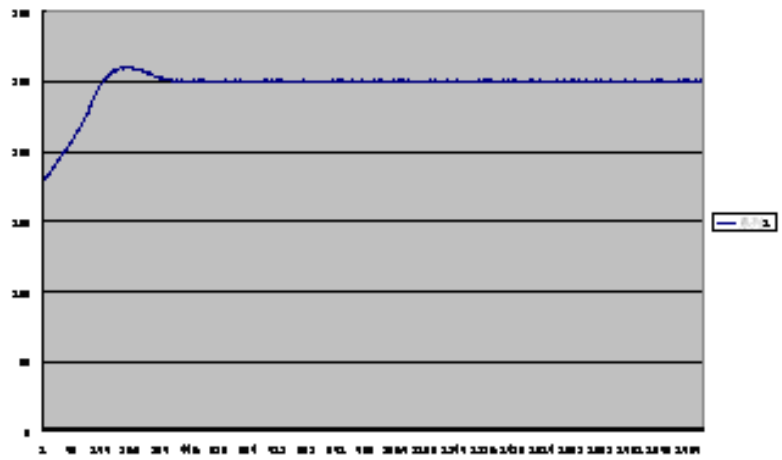

Fig.10 the code value of the received signal is 250 , the response curve of the motor after revise

\section{Conclusion}

Substituting PC104 with DSP is the development trend in the future for improving the tracking precision and the response due to the levity of the moving target. Selecting TMS320F2812 DSP as the servo controller is due to its high-speed performance and low power consumption. Besides the controller circuit is concise and reliable and the application is proved effective.

\section{References:}

[1]Su KuiFeng ,Theory and exploiture of TMS320F2812[M ],The electronic industry Publishing Company,2004

[2] Hu ShouSong,Theory of Autocontrol[M],Industry of National Defence Publishing Company, 1994.5

[3]Wang Yi Wei ZhongHe,Method for Compensating Speed Error and Acceleration Error of Servo System[J],1978.3-13-20

[4]Ma JiaGuang,Theory and Application for Compound [J],Control and Equivalent Compound Control,1988.5-1-15

[5]Wang JianLi,Study on TV Tracking System of O-E Theodolite to Track and Acquire Fast Moving Targets[D],2002.4 